\title{
Epidemiological traits of the malaria-like parasite Polychromophilus murinus in the Daubenton's bat Myotis daubentonii
}

\author{
Fardo Witsenburg", Franziska Schneider and Philippe Christe
}

\begin{abstract}
Background: The great diversity of bat haemosporidians is being uncovered with the help of molecular tools. Yet most of these studies provide only snapshots in time of the parasites discovered. Polychromophilus murinus, a malaria-like blood parasite, specialised on temperate-zone bats is a species that is being 'rediscovered'. This study describes the infection dynamics over time and between host sex and age classes.

Methods: For three years we followed the members of three breeding colonies of Myotis daubentonii in Western Switzerland and screened them for the prevalence and parasitemia of P. murinus using both molecular tools and traditional microscopy. In order to identify more susceptible classes of hosts, we measured, sexed and aged all individuals. During one year, we additionally measured body temperature and haematocrit values.

Results: Juvenile bats demonstrated much higher parasitemia than any other age class sampled, suggesting that first exposure to the parasite is very early in life during which infections are also at their most intense. Moreover, in subadults there was a clear negative correlation between body condition and intensity of infection, whereas a weak positive correlation was observed in adults. Neither body temperature, nor haematocrit, two proxies used for pathology, could be linked to intensities of infection.

Conclusion: If both weaker condition and younger age are associated with higher infection intensity, then the highest selection pressure exerted by $P$. murinus should be at the juvenile stage. Confusion over the identities and nomenclature of malarial-like parasites requires that molecular barcodes are coupled to accurate morphological descriptions.
\end{abstract}

Keywords: Polychromophilus, Myotis, Bats, Apicomplexa, Haemosporida, Susceptibility, Chiroptera

\section{Background}

Bats are the hosts to a large array of blood parasites (e.g. Bartonella, piroplasms, trypanosomes and microfillaria). In particular, bats are host to a unique collection of Haemosporida (Apicomplexa). Besides Plasmodium spp. and the rarer Hepatocystis spp., which infect several orders of Mammalia, bats host at least two unique genera not found outside the Chiroptera [1], Nycteria and Polychromophilus, plus two additional genera known from a single record (Dionisia [2] and Biguetellia [3]). The diversity of haemosporidian parasites of bats suggests, together with a recent phylogeny [4], that the Chiroptera

\footnotetext{
* Correspondence: fardo.witsenburg@unil.ch

Department of Ecology and Evolution, University of Lausanne, Biophore, $\mathrm{CH}-1015$, Lausanne, Switzerland
}

\section{Biomed Central}

might have been the original host of all mammalian Haemosporida.

Naturally, this has sparked renewed interest in bat haemosporidians, and Polychromophilus spp. have seen a particular rise in attention. The increasing ease and efficiency of molecular techniques has facilitated the detection of these parasites, enabling studies on its prevalence [5,6], phylogenetic origins [7] and the discovery of new species [8]. However its development in a host population both through time and between host classes and other ecological measures are lacking, keeping large parts of its biology still a mystery. The aim of the current study is to describe these parameters for a temperate-zone member of the Polychromophilus genus, P. murinus. Its type host is Vespertilio murinus, but an important reservoir species seems to be Myotis daubentonii, the 
Daubenton's bat, a common bat species spread across the Palearctic $[1,6,9]$.

Though its epidemiology is practically unknown, the life cycle of $P$. murinus is surprisingly well described, both in the host as well as the vector. Exoerythrocytic schizogony initially takes place in several organs, but finally develops inside the Kupffer cells of the liver $[1,10]$. The gametocytes are the only stages invading erythrocytes and these are taken up by the bat fly Nycteribia kolenatii (Nycteribiidae: Diptera). Here sexual reproduction takes place, after which an oocyst develops sporozoites on the gut wall [11].

The distribution of P. murinus has been documented in very few studies but since it has been observed in Italy, Switzerland and Great Britain $[6,11,12]$ it is likely that $P$. murinus has established itself in populations of $M$. daubentonii across Europe. In contrast, we have little idea about the prevalence variation over time, as well as the development of infection over the summer season. The aim of the current study was therefore to determine these epidemiological parameters in $M$. daubentonii at a local site. Secondly, by observing the susceptibility of each host age and sex class, the most likely source of the epidemic can be identified, which provides clues how the infection is maintained at this site. We will use both molecular methods, for parasite detection, and microscopy, for visual confirmation and assessment of the intensity of infection.

By definition, a parasite should have a negative effect on the host's fitness. The direct effects of an infection with a haemosporidian parasite can be very severe $[1,13]$, but may depend heavily on both the parasite and host species [14]. The only attempt at studying the physiological effects of $P$. murinus on bats failed when the artificially inoculated heterospecific host appeared unsusceptible to infection [15]. The attempt to infect a human with $P$. murinus by injecting infected bat blood failed as well; though the test subject did develop a fever, this was probably caused by other pathogens introduced by the injection [1]. Our aim here is not to perform a clinical experiment, but to explore possible effects of $P$. murinus infection using two physiological characteristics that can be used as proxies for pathology: haematocrit for anaemia and body temperature for fever.

\section{Methods}

\section{Sample collection}

Myotis daubentonii were captured during the seasons of 2010, 2011 and 2012 on the University of Lausanne campus, near Lake Geneva in Switzerland. The bats originated from three breeding colonies located in the forest on campus and were caught using a harp trap positioned over the Sorge stream at dusk. While highly pregnant and lactating females were immediately released upon capture, all other bats were used for sampling. Each bat was ringed to prevent resampling. The age of the bat, either 'adult' or 'subadult' was determined by the presence of a dark spot on the lower lip, which fades after 1-2 years. Each bat's forearm length (to the nearest $0.1 \mathrm{~mm}$ ) and weight (to the nearest 0.1 gram) were measured. We used the residuals from an OLS regression of body mass on forearm length as a measure of body condition [16]. In 2012, the haematocrit value (see below) and body temperature were also measured. Captured bats were immediately removed from the trap and body temperature was measured by inserting a lubricated probe into the rectum (RET-3 animal rectal probe and BAT-12 microprobe thermometer, Physitemp, Clifton, USA).

Blood was obtained by puncturing the uropatagial vein with a $0.5 \mathrm{~mm}$ gauge needle (Neolus). Between 5 and $30 \mu \mathrm{L}$ of blood were collected using either microvettes with EDTA (Sarstedt; seasons 2010/2011) or heparinized glass microcapillary tubes (Marcel Blanc \& Cie; season 2012). Samples were stored at $-20^{\circ} \mathrm{C}$ until molecular analysis. Haematocrit was measured by centrifuging the microcapillary tubes containing fresh blood for 7 minutes at $12,800 \mathrm{rpm}$. The haematocrit value was calculated by dividing the length of the red blood cell column by the total length (measured to the nearest $0.1 \mathrm{~mm}$ ).

After blood sampling, haemostatic cotton was applied to the punctured vein until bleeding ceased. All bats were captured under the licenses \#1317 and \#1656, authorized by the Cantonal Veterinarian Service of Vaud, Switzerland.

\section{Blood parasite observation}

From each blood sample, one drop of fresh blood was applied to a microscope object glass to make a thin smear. Slides were subsequently dried and immediately submerged in $100 \%$ methanol for fixation. Finally, a 5\% Giemsa-stain was applied for one hour to stain the cells. Slides were inspected with a light microscope at $600 \times$ and $1000 \times$ magnification. Polychromophilus murinus gametocytes were identified following Garnham [1] and Landau et al. [17]. Of a subset of gametocytes we noted the sex, counted manually the number of pigment granules and measured the dimensions of cells and nuclei.

The relative abundance of $P$. murinus gametocytes in the blood was estimated by scoring the number of $P$. murinus gametocytes observed in each smear at $600 \times$ magnification for 15 minutes. This measure, from now on referred to as parasitemia, should give an idea of the intensity of infection.

\section{Molecular analysis}

DNA was extracted using the Blood and Tissue spin column kit (Qiagen, CA), following the manufacturer's tissue protocol, with an overnight digestion and eluted in $2 * 50 \mu \mathrm{L}$. To control for contamination, a negative control 
was always included during the extraction process. The infection status of the bat host was determined by detecting the presence of a $705 \mathrm{bp}$ fragment of the mitochondrial cytochrome $b$ gene (cyt $b$ ) of the parasite following a nested PCR protocol. Primers, reagent concentrations and thermal profiles can be found in Megali et al. [6]. Amplified fragments were run on a $1.5 \%$ agarose gel and visualised under UV light. For every eight samples tested, a negative control was included in the amplification protocol. Each sample was tested in two independent tests. Samples with ambiguous results were tested again in duplicate. Samples remaining ambiguous were discarded from the data set.

A previous study at this site had demonstrated that the local $M$. daubentonii bats were exclusively infected by $P$. murinus and that this parasite population consisted of seven, almost identical cyt $b$ haplotypes [6]. The aim of our current study was not to describe the parasite molecularly. However, to confirm these previous findings, as well as our microscopic observations, we had a subset of the PCR positive samples purified and sequenced by a commercial agent (Microsynth, Switzerland) using both the forward and reverse primers of the nested reactions.

\section{Statistical analyses}

Factors influencing $P$. murinus prevalence, as assessed by the nested PCR protocol, were identified by logistic regression. The original model included the variables sex, age, body length and condition, as well as year and date, the latter variable expressed as standardized days since April $1^{\text {st }}$. Interactions were included based on their biological relevance: year $x$ date, date $x$ condition, sex $x$ condition and age $x$ condition. Terms were removed by backward selection, based on AIC and non-significant residual deviances until a minimal adequate model was found.

Since the parasitemia data was zero-inflated and showed signs of overdispersion, a zero-inflated (i.e. 'mixed') negative binomial approach (ZINB) was implemented using the pscl package for $\mathrm{R}$ [18]. To identify factors that influence $P$. murinus parasitemia in wild $M$. daubentonii hosts, this model considers the overly abundant zeros to come from two different processes, i.e. 'false zeros', caused either by poor observations or individuals that did not encounter the parasite, and 'true zeros', resulting from the covariates being unfavorable for the parasite [19]. For both the false-zero portion of the model, which attempts to discriminate between the two types of zeros, and the count portion, the same host-related covariates were used as for the previously described logistic regression of infection status. However, the variable date was also included in its squared form after graphical inspection of the data. A backward selection procedure was adopted wherein a term was dropped from either the false-zero or count portion of the model until no further decrease in AICc was observed. Each progressive model was tested for a significant change in log likelihood compared to its predecessor [19].

Juveniles were collected only in September and October of 2012. These are fledged young-of-the-year, that can be recognized by the incomplete ossification of their finger joints. Because of their age, it is not recommended to capture them earlier in the season and they were therefore not included in the previously described analyses. A comparison between juveniles and the other two age classes was therefore done separately. An F-value was obtained by performing an ANOVA on parasitemia by age class, with date as a covariate. This F-value was compared to a null distribution obtained by randomizing age 999 times.

The effects of parasitemia on bat hematocrit values and body temperatures were tested by linear regression. To linearize the relationship, parasitemia was $\log (x+0.5)$ transformed. As female mammals can have lower hematocrit levels [20] and higher body temperatures [21], sex was included as a cofactor in each analysis.

All statistical analyses were calculated using $\mathrm{R}$ ver. 3.0.1 [22].

\section{Results}

P. murinus gametocytes were detected in 58 blood smears (Figure 1). Gametocytes were large (Table 1), round to oval shaped, with a maximal diameter concurring with the described 6-8 $\mu \mathrm{m}$ [1]. Mature gametocytes would practically fill up the host cell, leaving mainly the edge of the host cell visible. This follows the description of Garnham [1], yet Landau et al. [17] mention that they do not completely fill up the host cell, but how much of the host lumen remains is not mentioned. The pigments are mouse-dropping shaped and of an irregular size, irregularly distributed across the cell; these clusters of pigments made counting them an ambiguous task and pigment numbers (Table 1) should therefore be taken with due caution.

The female macro- and male microgametocytes are easily distinguished, with the macrogametocyte having a compact, round, well-bordered nucleus in a dense cytoplasm. In contrast, the often more oval-shaped microgametocyte has a much lighter coloured cytoplasm with a diffuse, ill-defined and randomly shaped nucleus, which is considered a clear characteristic of $P$. murinus. Due to its irregular shape, measuring the male nucleus width in a consistent way was challenging and the values should again be taken with caution (Table 1).

Of the $212 \mathrm{M}$. daubentonii tested, 157 (74.1\%) were PCR positive for the cyt $b$ fragment. Of the 186 individuals of which also the blood smear had been searched for parasites, 58 (31.1\%) were positive. 80 individuals were negative by microscopic analysis but positive by PCR and 48 were negative according to both methods. All nine successfully 


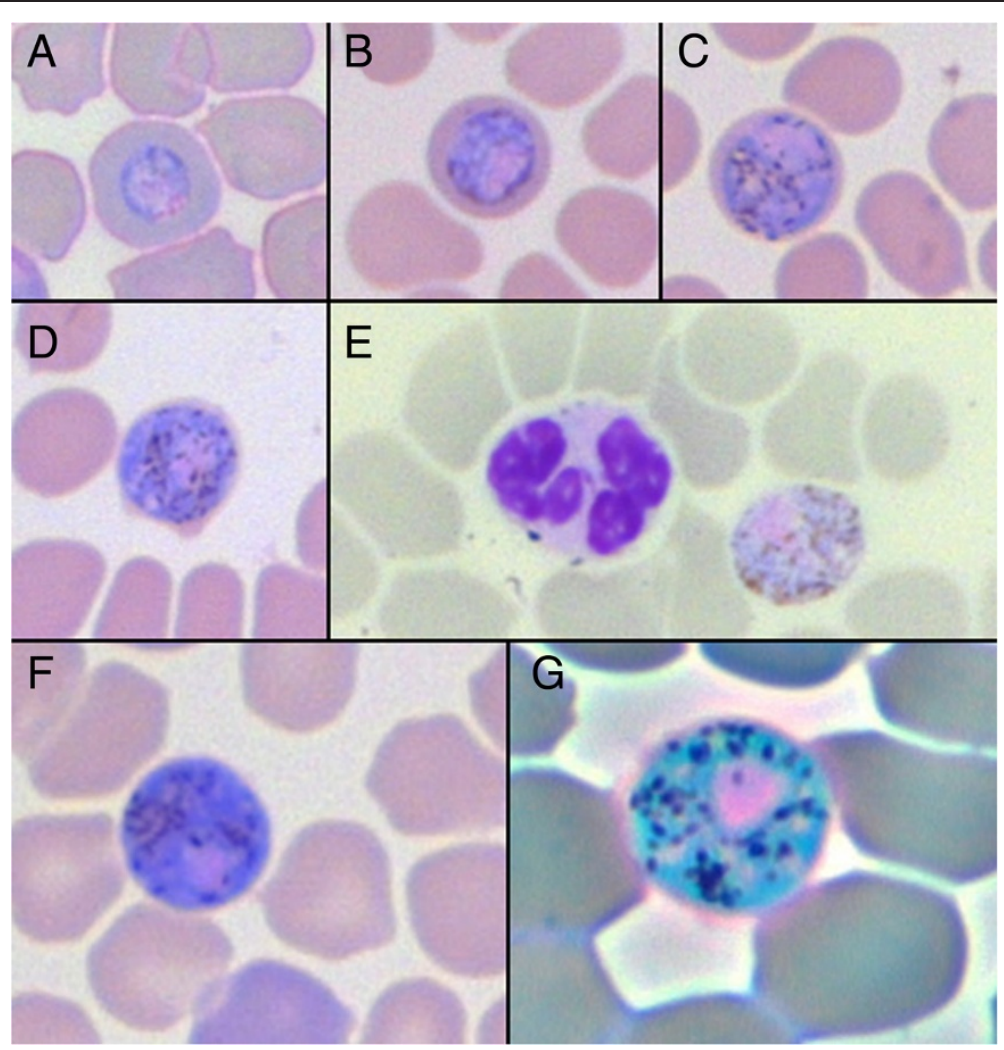

Figure 1 Images of Polychromophilus murinus gametocytes in Myotis daubentonii erythrocytes. (A-D) Different maturation stages of a gametocyte; (E) Mature male, microgametocyte with leucocyte; (F) Mature female, macrogametocyte; (G) Mature female, macrogametocyte, phase-contrast filter. Images A-F 600x magnification, image G 1000x magnification.

sequenced cytb fragments had a $100 \%$ identity with P. murinus, six were identical with haplotype 1 (Genbank Accession HM055583) and the three remaining with haplotype 2 (Genbank Accession HM055584; following [6]).

All required data for logistic regression analyses were available for 193 bats. Infection rates increased over the season, but only in 2010 a period of peak infection appeared, around July-August (Figure 2A); other years showing either an unsteady increase (2011) or a flat trend (2012). The final model of $P$. murinus prevalence contained both date, year and its interaction (Table 2). All other variables were retained as well, but not their interactions or age (Table S1 in Additional file 1). The goodness-of-fit test was not significant (HosmerLemeshow, $X^{2}=8.558, p=0.38$ ), but visually many variables showed no pattern at all. Many intercorrelations existed between the predictors, which can make GLM solutions very sensitive to small variations in predictors [23]. To assess the robustness of our solution, we randomly split the data in two and three subsets, each subset containing respectively $50 \%$ or $33 \%$ of all observations, and used these to retest our model. Upon retesting, many variables disappeared from the model. Only date appeared as a reliable predictor and, with the exception of one

Table 1 Morphometric measurements taken of the gametocytes, in $\mu \mathrm{m}$

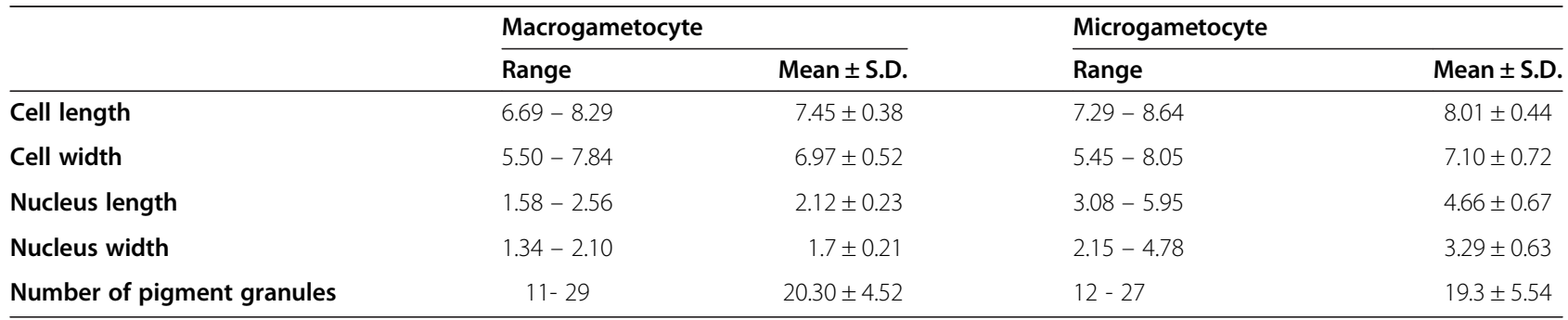

The slightly smaller female macrogametocyte $(n=26)$ had a clearly defined compact nucleus, whereas the nucleus of the male microgametocyte $(n=16)$ was large and diffuse with ill-defined borders. 

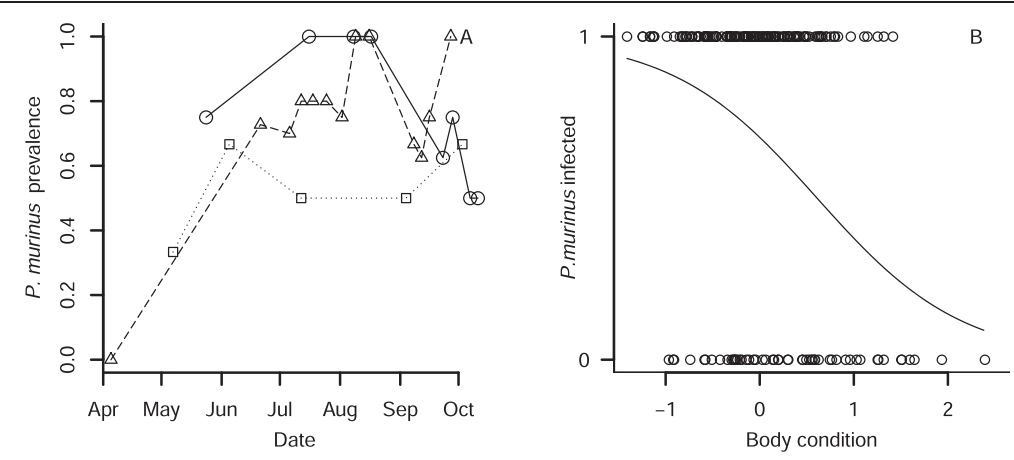

Figure 2 Results from the logistic regression. (A) Prevalence of $P$. murinus in local M. daubentonii through the season, separated by year. Circles and continuous line: 2010; triangles and dashed line: 2011; squares and dotted line: 2012. (B) The possibility of infection with P. murinus reduces with increased condition of the bat.

model, body condition as well (Figure 2B; Table S3 in Additional file 1).

Parasitemia data were collected from a total of 186 M. daubentonii for the analysis of blood parasite abundances. The minimal adequate ZINB model retained multiple terms in the count model, but none in the false-zero portion (Table 3, Table S2 in Additional file 1), though the difference between the last two models (with or without age in the false-zero part of the model $)$ was only marginal $(\triangle \mathrm{AICc}=0.58$; Table $\mathrm{S} 2$ in Additional file 1). The count portion of the ZINB model retained several variables. Condition and age had a significant interaction. In adults, parasitemia increased slightly with increasing body condition, whereas in subadults a strong negative relationship existed between parasitemia and body condition (Table 3, Figure 3A). In general, individuals in higher body condition had lower parasitemia, and the interaction with date indicated that peak parasitemia was reached sooner in bats in high body condition (Figure 3B). Though ranges overlapped, juveniles had higher median and maximum parasitemia by one and two orders of magnitude respectively (Figure 4, randomized F-test: $n=23$, randomizations $=999, p=0.014$ ).

Parasitemia of $P$. murinus had no effect on the body temperature of the bats when corrected for sex (multiple

Table 2 Estimates of the parameters and their significance for the logistic regression of prevalence

\begin{tabular}{|c|c|c|c|c|}
\hline Parameter & Estimate & Standard error & $x^{2}$ & $p$ \\
\hline Year (2011) & -0.783 & 0.545 & - & - \\
\hline Year (2012) & -1.996 & 0.650 & - & - \\
\hline Date & -0.247 & 0.417 & - & - \\
\hline Sex (male) & -1.061 & 0.471 & 5.32 & 0.021 \\
\hline Condition & -1.298 & 0.316 & 20.01 & $<1^{*} 10^{-5}$ \\
\hline Length & -0.326 & 0.183 & 3.32 & 0.068 \\
\hline Date x year (2011) & 1.045 & 0.506 & 6.36 & 0.041 \\
\hline Date x year (2012) & 0.176 & 0.540 & $"$ & $"$ \\
\hline
\end{tabular}

linear regression: $F_{2,39}=0.057 ; p=0.943$; Figure 5A). Hematocrit value did not appear to be influenced by the abundance of gametocytes either (multiple linear regression: $F_{2,44}=0.078 ; p=0.463$; Figure $5 \mathrm{~B}$ ).

\section{Discussion}

Polychromophilus murinus reached its highest abundances in juvenile $M$. daubentonii, which have to carry the heaviest burden of infection. However, no direct physiological effect of infection was found. Furthermore, the zero-inflated model showed that across the whole season parasitemia was much higher in subadults than adults. This suggests that with age, bats are better able to cope with the infection.

Previous work has shown that the primary exposure to a haemosporidian parasite causes much higher parasitemia in hosts than any further encounters [1]. Our study demonstrated much higher parasite abundances in juveniles than other age classes, supporting this observation. Bat ectoparasites synchronise their reproduction with their hosts [24]. After female bats have given birth, the ectoparasites move in mass onto the pups [25], introducing blood parasites to the neonates at a very early stage of development. The primary exposure to the haemosporidian parasite

Table 3 Estimates of the parasitemia statistical model parameters and their significance for the model

\begin{tabular}{lcccc}
\hline Parameter & Estimate & Standard error & $\boldsymbol{X}^{\mathbf{2}}$ & $\boldsymbol{p}$ \\
\hline Count model $\boldsymbol{\mu}$ & & & & \\
Date $^{2}$ & -0.472 & 0.178 & 5.62 & 0.018 \\
Date & 0.189 & 0.189 & - & - \\
Condition & 0.396 & 0.525 & - & - \\
Age $($ subadult) & 1.144 & 0.381 & - & - \\
Date $\times$ condition & -1.130 & 0.368 & 9.77 & 0.002 \\
Age $\times$ condition & -1.778 & 0.679 & 6.31 & 0.012 \\
False zero model $\boldsymbol{\pi}$ & & & & \\
None & - & - & - & - \\
\hline
\end{tabular}



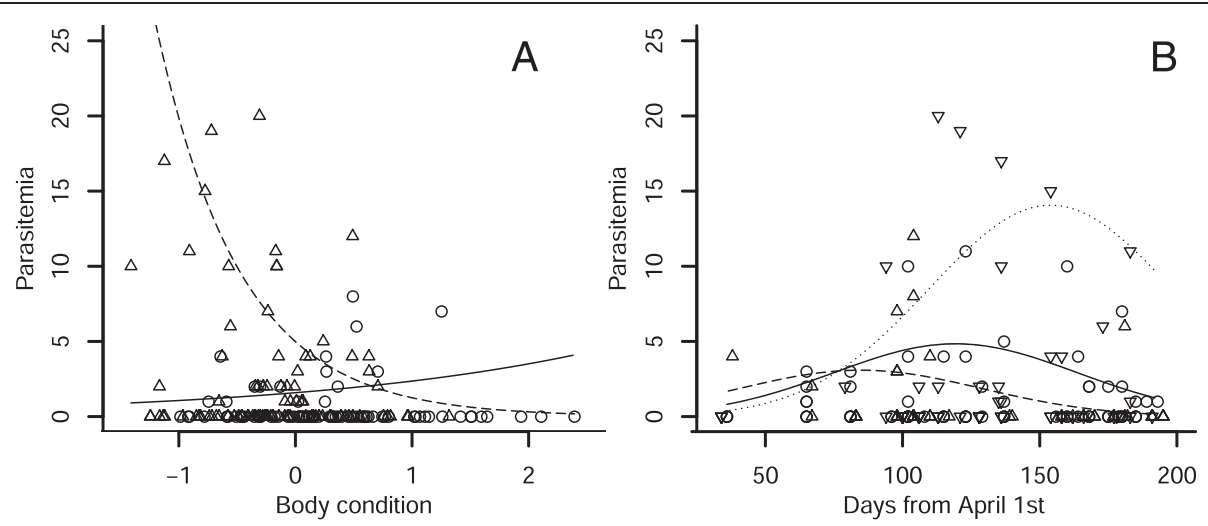

Figure 3 Predictors of the intensity of infection as approximated by parasitemia. (A) The relation between body condition and parasitemia differs between age classes. Circles and continuous trend line: adults; triangles and dashed trend line: subadults. (B) Parasitemia changes through the season and interacts with body condition. Continuous trend line and circles: bats with mean body condition; dashed trend line and up-facing triangles: bats in high body condition (>mean +0.5 s.d.); dotted trend lines and down-facing triangles: bats in low body condition ( $<$ mean - 0.5 s.d.).

causes extreme high levels of parasitemia, which in turn increases the probability of establishing an infection in the abundant newly emerged bat flies. As bat flies are long lived and can overwinter [15], this in itself might suffice to maintain the $P$. murinus infection.

The bat's body condition was linked to the chance of being infected and it correlated with the progression of infection as well as the maximum intensity of the infection. Notably, the effect of host body condition on the parasite intensity depended on the age of the host, for which several non-mutually exclusive processes might be responsible. Subadults with larger fat storages could be better equipped to mount a costly immune response [26], though no direct relation was found in the Brazilian

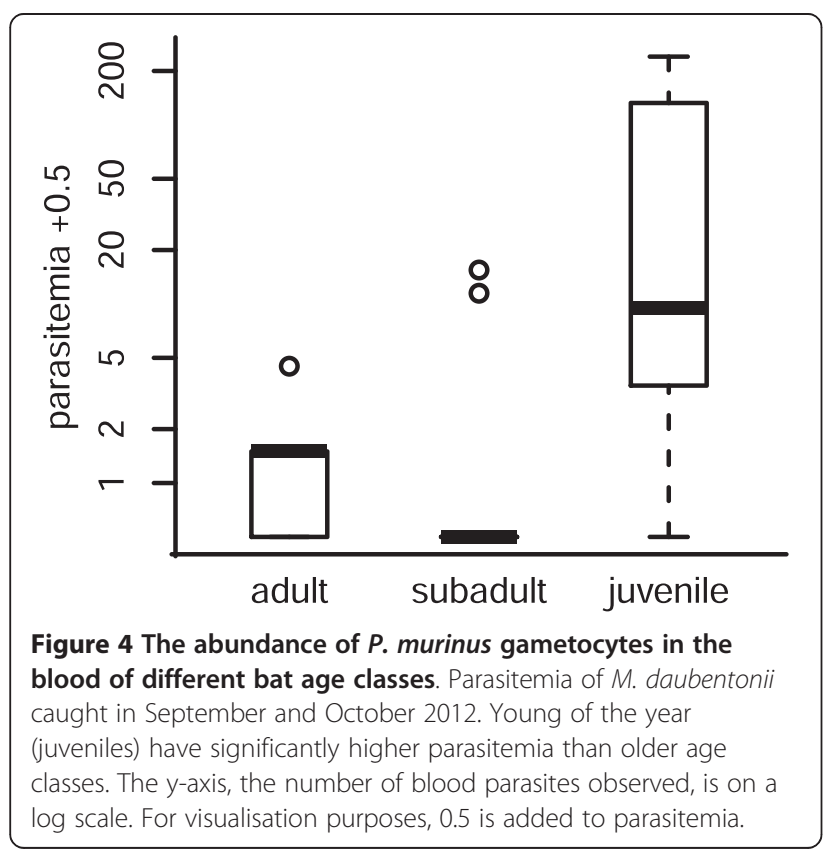

free-tailed bat Tadarida brasiliensis [27]. In M. daubentonii body condition increases with maturation [28]. The strong decline of parasitemia with condition seen in subadults might therefore actually represent maturing individuals which at the same time learn to cope with infection.

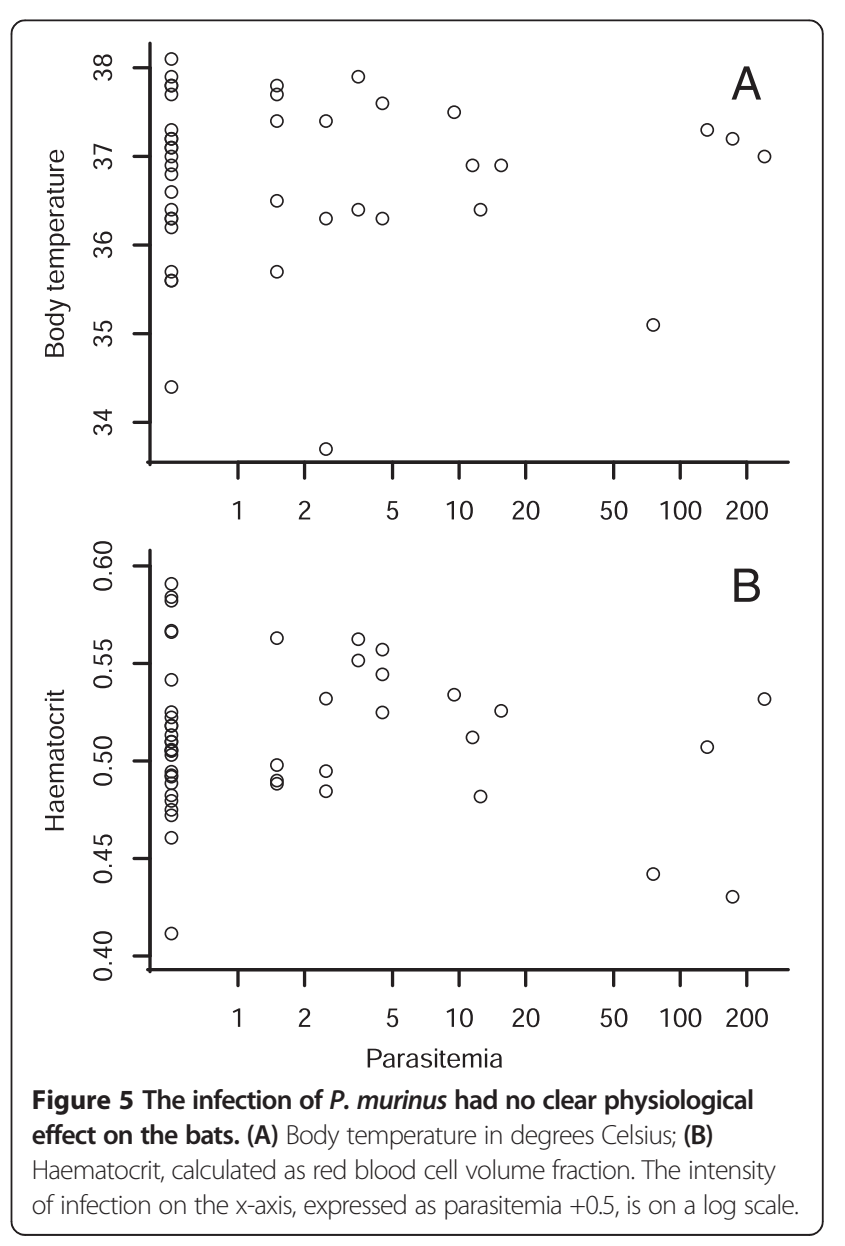


However, in adults, the relationship between condition and parasitemia is slightly positive. Heavier adults might be trading off mass against immunity, though it is unclear why this would only affect adults [26]. Like other bat ectoparasites [29], the vector $N$. kolenatii is attracted to hosts in higher body condition [30]; these bats might therefore be more often exposed to new infections, which causes a slight increase in parasitemia. It might also be a sampling artefact: perhaps for bats with similar levels of infection, only those in good condition can tolerate it enough to go foraging at night when we caught them. Lastly, the positive correlation might be caused by pregnant females, which are relatively heavy for their size and also immunosuppressed [25]. Though highly gravid females were never sampled, females in earlier stages of gestation are more difficult to recognize and might therefore be present in the data set.

It is worth noting, that in our statistical analyses we have considered a bat's body condition as one of the causes predicting the likelihood of infection and its intensity. Yet, in contrast to date or age, the reverse is just as likely. Mounting an immune response requires energy which should reduce fat reserves [26]. Loss of body mass might therefore very well be a symptom of infection with $P$. murinus. Only experimental infections under controlled conditions could resolve this question of cause and effect.

Apart from possible weight loss, we found no other sign of pathology. Bats showed no signs of fever; however, whether or not fever is applied by heterotherms is debated [31]. The lack of anaemia on the other hand might be because of the biology of $P$. murinus. Unlike Plasmodium spp., Polychromophilus spp. have no asexual multiplication in the blood. The number of erythrocytes destroyed during an infection should therefore be much lower compared to other malaria species.

Over the three years we found an average infection rate of $74.1 \%$ which is the same rate as Megali et al. [6] found at the same site in 2009 using the same screening method. Prevalence was not stable throughout the season, but this pattern seemed different each year suggesting either random emergent fluctuations prevalence or the influence of (unmeasured) climatic variables.

Prevalence was much higher based on PCR than based on the microscopy results. It is well established that nested PCR is more sensitive than pure visual control, though the two methods can approach each other in efficacy [32]. The proportion of 'false zeros' determined by the ZINB model did not correspond to the number of bats tested negative by PCR. When all PCR-negative individuals were removed from the parasitemia analysis, zero-inflation was still an issue. The false zeroes were therefore not only caused by hosts that had not encountered the parasite, but also 'bad observations'. In our case, the ZINB found no factor influencing the appearance of false zeroes, indicating that this rate of zero detection was unbiased across the categories of hosts. In contrast with this, the actual probability of being infected, as demonstrated by the logistic regression, depends mainly on time of season and the host's condition.

The rise of molecular techniques in parasitology also inadvertently meant a decline of light microscopy [33], not only to describe new parasite species or lineages (but see: [34]), but also to determine parasite prevalence and abundance. Although PCR and qPCR are undeniably much more efficient approaches for analysing hundreds of blood samples at a time, some pitfalls are associated with the use of molecular techniques. Primers may be unsuitable for the parasite present [33] or only dead-end stages, injected by the vector, are detected [35], leading to the false conclusions that the host is uninfected or infected respectively. In this study we used PCR methods to scan for infections. Our main findings, however, stem from our results produced by microscopical investigation.

The currently available species descriptions for $P$. murinus are either not very exhaustive [1] or contradictory; Landau et al., for example, would sometimes describe the P. murinus gametocytes as of the type malariae [2], yet in other publications as of the type falciparum [3], whereas Garnham describes it as of type vivax [1]. Here we tried to provide some quantitative measures with our morphological description of $P$. murinus to link the morphospecies to the available genetic sequences. With the current confusing species descriptions available, a proper redescription would be welcome. However, for a complete description it is essential to have access to the schizonts, which can be sampled by dissection. Such complete redescriptions coupled to sound genetic bar codes is needed to resolve the presently confused state of taxonomy among malarial-like parasites.

\section{Conclusion}

Juvenile $M$. daubentonii clearly carried the highest $P$. murinus parasite loads. Furthermore, bat host condition was strongly correlated to the infection intensity. If both weaker condition and younger age are associated with a more intense infection, then the highest selection pressure exerted by $P$. murinus should be on the juvenile $M$. daubentonii. Weak young are pruned from the population and any surviving bats will have developed a tolerance against further infections with $P$. murinus. This could explain both the absence of any pathological symptoms in adults as well as the ability of the parasite to remain present in bat populations throughout the year. 


\section{Additional file}

Additional file 1: The file contains supplementary results of the statistical analyses. S1) Table of the backward-selection procedure of the prevalence model. S2) Table of the backward-selection procedure of the parasitemia model. S3) Stability of the backwards selection procedure, by using subsets of the prevalence data set.

\section{Competing interests}

The authors declare that they have no competing interests.

\section{Authors' contributions}

FW conceived the study, collected data, performed the molecular and statistical analyses and wrote the final manuscript. FS participated in data collection, parasite detection by microscopy and PCR, performed an initial data analysis and wrote an earlier version of this manuscript. PC conceived the study, interpreted the results and contributed to the final manuscript. All authors read and approved the final version of the manuscript.

\section{Acknowledgements}

The authors would like to thank all those who helped catching bats in the field, in particular Ludovic Dutoit and Laura Clément. We are also grateful to Laura for DNA sequencing of the samples. Jérôme Goudet gave helpful statistical advice. We thank the Centre de Coordination Ouest pour l'Etude et la Protection des Chauves-souris in Geneva for logistic support. We thank Tania Jenkins, Karen McCoy, Nadir Alvarez and an anonymous reviewer for helpful comments on earlier drafts of this paper. This study has been supported by grants no 31003A-120479 and 31003A-138187 by the Swiss National Science Foundation.

Received: 18 June 2014 Accepted: 24 November 2014

Published online: 09 December 2014

\section{References}

1. Garnham PCC: Malaria parasites and other Haemosporidia. 1st edition. Oxford: Blackwell Scientific Publications; 1966.

2. Landau I, Chabaud AG, Miltgen F, Baccam D: Dionisia bunoi n. gen. n. sp. Haemoproteidae parasite du microchiroptère Hipposideros cyclops au Gabon. Ann Parasitol Hum Comp 1980, 55:271-280.

3. Landau I, Baccam D, Ratanaworabhan N, Yenbutra S, Boulard Y, Chabaud AG: Nouveaux Haemoproteidae parasites de chiroptères en Thailande. Ann Parasitol Hum Comp 1984, 59:437-447.

4. Schaer J, Perkins SL, Decher J, Leendertz FH, Fahr J, Weber N, Matuschewski K High diversity of West African bat malaria parasites and a tight link with rodent Plasmodium taxa. Proc Natl Acad Sci U S A 2013, 110:17415-17419.

5. Concannon R, Wynn-Owen K, Simpson VR, Birtles RJ: Molecular characterization of haemoparasites infecting bats (Microchiroptera) in Cornwall, UK. Parasitology 2005, 131:489-496.

6. Megali A, Yannic G, Christe P: Disease in the dark: molecular characterization of Polychromophilus murinus in temperate zone bats revealed a worldwide distribution of this malaria-like disease. Mol Ecol 2011, 20:1039-1048.

7. Witsenburg F, Salamin N, Christe P: The evolutionary host switches of Polychromophilus: a multi-gene phylogeny of the bat malaria genus suggests a second invasion of mammals by a haemosporidian parasite. Malar J 2012, 11:53.

8. Duval L, Robert V, Csorba G, Hassanin A, Randrianarivelojosia M, Walston J, Nhim T, Goodman SM, Ariey F: Multiple host-switching of Haemosporidia parasites in bats. Malar J 2007, 6:157.

9. Garnham PCC: The zoogeography of Polychromophilus. Ann Parasitol Hum Comp 1973, 48:231-242.

10. Landau I, Rosin G, Miltgen F, Leger N, Mialhe E: La schizogony de Polychromophilus (Haemoproteidae) parasite cosmopolite de Microchiroptère. C R Acad SC Paris D 1977, 285:1311-1313.

11. Gardner RA, Molyneux DH, Stebbings RE: Studies on the prevalence of Hematozoa of British bats. Mammal Rev 1987, 17:75-80.

12. Corradetti A: Alcuni protozoi parassiti di Nycteribiidae del genere Listropoda. Ann Igiene 1936, 46:444-460.

13. Valkiũnas G: Avian Malaria Parasites and Other Haemosporidia. English edition. Boca Raton: CRC Press; 2005.
14. Palinauskas V, Valkiunas GN, Bolshakov CV, Bensch S: Plasmodium relictum (lineage P-SGS1): Effects on experimentally infected passerine birds. Exp Parasitol 2008, 120:372-380

15. Gardner RA, Molyneux DH: Polychromophilus murinus: a malarial parasite of bats: life-history and ultrastructural studies. Parasitology 1988 96:591-605.

16. Schulte-Hostedde Al, Zinner B, Millar JS, Hickling GJ: Restitution of mass-size residuals: validating body condition indices. Ecology 2005, 86:155-163.

17. Landau I, Rosin G, Miltgen F, Hugot JP, Leger N, Beveridge I, Baccam D: Sur le genre Polychromophilus (Haemoproteidae, parasite de microchiroptères). Ann Parasitol Hum Comp 1980, 55:13-32.

18. Zeileis A, Kleiber C, Jackman S: Regression models for count data in R. J Stat Softw 2008, 27:1-25.

19. Zuur AF, leno EN, Walker NJ, Saveliev AA, Smith GM: Mixed Effects Models and Extensions in Ecology With R. New York: Springer; 2009.

20. Nemeth N, Kiss F, Furka I, Miko I: Gender differences of blood rheological parameters in laboratory animals. Clin Hemorheol Microcirc 2010, 45:263-272.

21. Cryan PM, Wolf BO: Sex differences in the thermoregulation and evaporative water loss of a heterothermic bat, Lasiurus cinereus, during its spring migration. J Exp Biol 2003, 206:3381-3390.

22. R Core Team: R: A Language and Environment for Statistical Computing. Vienna, Austria: R Foundation for Statistical Computing; 2012.

23. Quinn GP, Keough MJ: Experimental Design and Data Analysis for Biologists. Cambridge: Cambridge University Press; 2002.

24. Lourenço S, Palmeirim JM: Which factors regulate the reproduction of ectoparasites of temperate-zone cave-dwelling bats? Parasitol Res 2008, 104:127-134.

25. Christe P, Arlettaz R, Vogel P: Variation in intensity of a parasitic mite (Spinturnix myoti) in relation to the reproductive cycle and immunocompetence of its bat host (Myotis myotis). Ecol Lett 2000, 3:207-212.

26. Lochmiller RL, Deerenberg C: Trade-offs in evolutionary immunology: just what is the cost of immunity? Oikos 2000, 88:87-98.

27. Allen LC, Turmelle AS, Mendonca MT, Navara KJ, Kunz TH, McCracken GF: Roosting ecology and variation in adaptive and innate immune system function in the Brazilian free-tailed bat (Tadarida brasiliensis). J Comp Physiol B 2009, 179:315-323.

28. Encarnacao JA, Kierdorf $U$, Ekschmitt $K$, Wolters V: Age-related variation in physical and reproductive condition of male Daubenton's bats (Myotis daubentonii). J Mammal 2006, 87:93-96.

29. Christe P, Giorgi MS, Vogel P, Arlettaz R: Differential species-specific ectoparasitic mite intensities in two intimately coexisting sibling bat species: resource-mediated host attractiveness or parasite specialization? J Anim Ecol 2003, 72:866-872.

30. Witsenburg F, Schneider F, Christe P: Signs of a vector's adaptive choice: on the evasion of infectious hosts and parasite-induced mortality. Oikos 2014. doi:10.1111/oik.01785.

31. Canale $\mathrm{Cl}$, Henry P-Y: Energetic costs of the immune response and torpor use in a primate. Funct Ecol 2011, 25:557-565

32. Valkiunas G, lezhova TA, Krizanauskiene A, Palinauskas V, Sehgal RNM, Bensch S: A comparitive analysis of microscopy and PCR-based detection methods for blood parasites. J Parasitol 2008, 94:1395-1401.

33. Perkins SL, Martinsen ES, Falk BG: Do molecules matter more than morphology? Promises and pitfalls in parasites. Parasitology 2011, 138:1664-1674.

34. Palinauskas V, Kosarev V, Shapoval A, Bensch S, Valkiunas G: Comparison of mitochondrial cytochrome $b$ lineages and morphospecies of two avian malaria parasites of the subgenera Haemamoeba and Giovannolaia (Haemosporida : Plasmodiidae). Zootaxa 2007, 1626:39-50.

35. Valkiunas G, lezhova TA, Loiseau C, Sehgal RNM: Nested cytochrome b polymerase chain reaction diagnostics detect sporozoites of hemosporidian parasites in peripheral blood of naturally infected birds. J Parasitol 2009, 95:1512-1515.

\section{doi:10.1186/s13071-014-0566-7}

Cite this article as: Witsenburg et al:: Epidemiological traits of the malaria-like parasite Polychromophilus murinus in the Daubenton's bat Myotis daubentonii. Parasites \& Vectors 2014 7:566 